\title{
ON THE EDGE
}





\section{ON THE EDGE}

\section{LIFE ALONG THE RUSSIA-CHINA BORDER}

Franck Billé and Caroline Humphrey

HARVARD UNIVERSITY PRESS

Cambridge, Massachusetts \& London, England 2021 
Copyright (C) 2021 by the President and Fellows of Harvard College All rights reserved

Printed in the United States of America

First printing

Jacket photograph: Juliet Butler / Alamy Stock Photo

Jacket design: Annamarie McMahon Why

9780674269491 (EPUB)

9780674269538 (PDF)

The Library of Congress has cataloged the printed edition as follows:

Names: Billé, Franck, author. I Humphrey, Caroline, author.

Title: On the edge : life along the Russia-China border / Franck Billé and

Caroline Humphrey.

Description: Cambridge, Massachusetts : Harvard University Press, 2021. I

Includes bibliographical references and index.

Identifiers: LCCN 2021017869 | ISBN 9780674979482 (cloth)

Subjects: LCSH: Borderlands-Russia (Federation) I Borderlands-China. I

Boundaries-Anthropological aspects-Russia (Federation) I Boundaries-

Anthropological aspects_China. I Russia (Federation)—Boundaries-China. I

China-Boundaries-Russia (Federation)

Classification: LCC DK68.7.C6 B55 2021 I DDC 957/.7—dc23

LC record available at https://lccn.loc.gov/2021017869 
To our families 
